\title{
Ibuprofen Versus Indomethacin for Medical Closure of the Patent Arterial Duct: A Pooled Analysis by Route of Administration
}

\author{
Rohit Loomba ${ }^{1}$, Karan Nijhawan ${ }^{2}$ \\ 1. Department of Cardiology, Advocate children's hospital, chicago, USA 2. Medicine, Rush University \\ Medical Center
}

$\square$ Corresponding author: Rohit Loomba, loomba.rohit@gmail.com Disclosures can be found in Additional Information at the end of the article

\section{Abstract}

Introduction:

Preterm infants are at increased risk of having a patent arterial duct (PAD). PADs may cause congestive heart failure, respiratory distress, necrotizing enterocolitis, and renal impairment. Consequently, in some infants, it becomes necessary to attempt closure of the PAD. Surgical closure can be difficult in small infants and is not without its risks; thus, medical closure offers advantages. Cyclooxygenase inhibitors have been used for medical closure of the PAD with both ibuprofen and indomethacin having been used clinically.

\section{Methods:}

We performed a systematic review of the literature to identify all studies comparing ibuprofen and indomethacin. Studies comparing ibuprofen and indomethacin for closure of the PAD in premature infants were included in the meta-analysis. A subanalysis was performed to compare the route of administration. Efficacy endpoints studied were PAD closure and surgical ligation while adverse effects studied were death in the first month of life, necrotizing enterocolitis, gastrointestinal bleeding, intestinal perforation, bronchopulmonary dysplasia in the first month of life, Grade 3 or 4 intraventricular hemorrhage, and change in the serum creatinine after treatment.

\section{Results:}

Ibuprofen and indomethacin were equally effective in closing the PAD in premature infants and demonstrated no difference in the incidence of adverse events. In respect to the route of both drugs via the intravenous route, the only difference noted between the ibuprofen and indomethacin was that ibuprofen was associated with a lesser increase in serum creatinine after treatment.

\section{(C) Copyright 2015}

Loomba et al. This is an open access article distributed under the terms of the Creative Commons Attribution License CC-BY 3.0., which permits unrestricted use, distribution, and reproduction in any medium, provided the original author and source are credited.

\section{Conclusion:}

Ibuprofen and indomethacin are equally effective in PAD closure without any difference in the incidence of adverse events. Importantly, oral ibuprofen was as effective as intravenous indomethacin. 
Categories: Cardiology, Pediatrics

Keywords: patent arterial duct, patent ductus arteriosus, ibuprofen, ligation, premature, indomethacin

\section{Introduction}

The arterial duct (AD) is a vascular structure that is necessary for fetal life, allowing for much of the right ventricular output to be shunted from the pulmonary artery to the descending aorta, thereby allowing for the developing lungs to receive enough blood to sustain development. Oxygenation, however, occurs at the level of the placenta during fetal life, and thus, blood does not need to pass through the pulmonary vasculature itself. The $\mathrm{AD}$ closes shortly after birth. In infants born at 30 to 40 weeks gestational age, the AD closes by 72 hours in $90 \%$, and in infants born at greater than 40 weeks gestational age, the AD closes in $100 \%$ by 72 hours [1-3].

The $\mathrm{AD}$ is considered to be a patent ductus arteriosus (PAD) if it does not close by 72 hours of life. The prevalence of PAD is not entirely known but is present in approximately $0.06 \%$ of all live births with estimates of silent PADs having a prevalence of 0.1 to $0.2 \%$. The prevalence of PADs is greater in those with lower birth weight and preterm gestation. For infants weighing 1,000 to 1,500 grams, $25 \%$ will have a $\mathrm{PAD}, 70 \%$ of whom will require treatment, and for infants less than 1,000 grams, 65\% will have a PAD, $85 \%$ of whom will require treatment [4-7].

What justifies treatment is currently under debate with many advocating for less treatment to avoid potential adverse effects of either surgical ligation or medical therapy. Others advocate being aggressive with treatment to avoid potential adverse effects of the PAD [8-9].

If the decision is made to treat, medical therapy is an option for premature infants. Medical therapy used to consist of indomethacin; however, over the last few years, ibuprofen has become an increasingly popular alternative. Both are still used in various settings without a common practice. We present a pooled analysis of the efficacy and safety of ibuprofen and indomethacin for the closure of the PAD in preterm infants.

\section{Materials And Methods}

A systematic review of the literature was performed to identify manuscripts describing comparisons between ibuprofen and indomethacin for closure of the PAD. This was a newly conducted review, and no previous review protocol has been established for the effectiveness in closing the $\mathrm{AD}$ and the need for surgical ligation. The following adverse events were studied: death in the first month of life, necrotizing enterocolitis, gastrointestinal bleeding, intestinal perforation, bronchopulmonary dysplasia in the first month of life, Grade 3 or 4 intraventricular hemorrhage, and change in serum creatinine after attempted treatment.

\section{Manuscript search and identification strategy}

Manuscripts were identified using electronic databases, including PubMed, EMBASE, and Ovid, which were queried using either "ibuprofen" and/or "indomethacin" in combination with "ductus arteriosus" or "arterial duct". No specific restriction on year of publication was used. Studies in languages other than English were excluded. Resulting studies were then screened by title and abstract with manuscripts suspected of being relevant being retrieved in their entirety. References of these were hand searched for additional relevant manuscripts. No direct contact with manuscript authors was required to obtain full text manuscripts. Only studies with an ibuprofen and indomethacin group were included. Those with no data reported for any of the endpoints of interest or studies with data not deemed suitable for extraction were also excluded.

These full text manuscripts were then reviewed by two of the authors (RL, KN) and assessed for 
quality. Any disparities in scoring of manuscripts were then discussed and resolved. The Cochrane Handbook for Systematic Review of Interventions was used for quality evaluation. Published manuscripts available in full text were included in this review if they presented data comparing ibuprofen and indomethacin with respect to the outcomes listed above. Studies were included in this analysis if they included at least one of the outcomes identified above.

\section{Data extraction}

Data regarding baseline patient characteristics and identified outcomes were extracted from the manuscripts identified for inclusion. Trial level data were extracted independently with the use of a data collection form by two authors. The data extraction was then independently reviewed by another author to ensure the integrity of the resulting data. Authors of included studies were not contacted for additional data.

\section{Bias analysis}

Bias was assessed using the Cochrane Risk of Bias Tool. Specifically, patient eligibility, randomization and concealment of allocation, blinding, and completeness of outcome data were assessed using this scale. Publication bias was assessed qualitatively using forest plots for endpoints with 10 or more studies included.

\section{Data analysis}

Numeric data are presented as means with standard deviations. Categorical data are presented as frequencies with absolute numbers as well as percentages. Results are presented as pooled odds ratios (OR) with $95 \%$ confidence intervals (CI) or as mean difference (MD) where appropriate. Heterogeneity between studies was identified using chi-square and $\mathrm{I}^{2}$ tests. For outcomes with no significant heterogeneity present, a fixed effects model was used. A random effects model was used if either the $\mathrm{p}$-value was significant or the $\mathrm{I}^{2}$ statistics was greater than $50 \%$. P-values of $\leqslant 0.05$ were considered statistically significant. The Mantel-Haenszel method was used for dichotomous endpoints while mean difference was used for continuous endpoints. Meta-analysis and forest plot creation were done using RevMan 5.0 (Cochrane Collaboration, Oxford, UK).

Analysis was done separately by route of administration. All endpoints were analyzed in the comparison of intravenous (IV) ibuprofen and IV indomethacin, oral ibuprofen versus IV indomethacin, and oral ibuprofen versus oral indomethacin. Data for all endpoints was not available for all routes of administration.

\section{Sensitivity analyses}

Sensitivity analyses were conducted to determine the effect of study size, study weight, year of publication, and study design.

\section{Results}

\section{Search results}

A total of 723 manuscripts were identified with 407 remaining after duplicates were identified and removed. Titles and abstracts of these manuscripts were reviewed, and 87 manuscripts were identified for full-text review. Of the 320 manuscripts not advancing to full-text review, 270 were excluded as there was no comparison between ibuprofen and indomethacin and 50 were excluded because they were reviews, meta-analyses, case reports, series, or letters. After a full-text review of the 87 identified manuscripts, 26 studies were identified as comparing the use of ibuprofen and indomethacin for closure of the PAD in preterm infants. Four of these 


\section{Cureus}

studies contained data for none of the predefined endpoints of interest or had data not suitable for extraction, and thus, a total of 22 studies were included in the final pooled analysis (Figure 1) [10-31]. Of the included studies, 14 (64\%) were randomized while eight (36\%) were retrospective studies (Table 1).

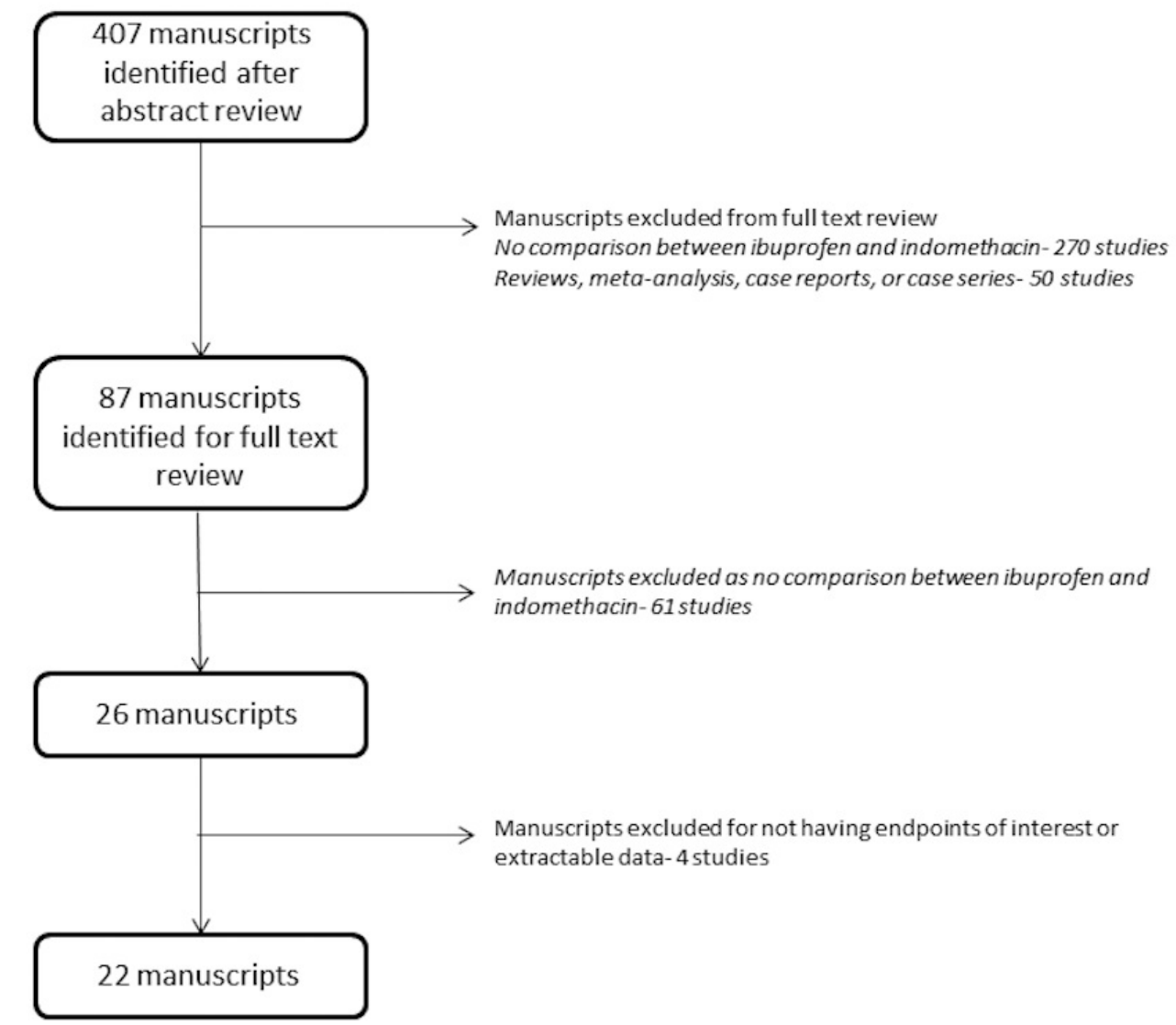

FIGURE 1: Study methodology

Study methodology

\begin{tabular}{|c|c|c|c|c|c|c|c|c|c|c|c|}
\hline \multirow[t]{2}{*}{ Study } & \multirow[t]{2}{*}{ Design } & \multicolumn{5}{|c|}{ Ibuprofen } & \multicolumn{5}{|c|}{ Indomethacin } \\
\hline & & $\mathrm{n}$ & Male & $\begin{array}{l}\text { Gestational age } \\
\text { (wks) }\end{array}$ & $\begin{array}{l}\text { Birth weight } \\
(\mathrm{kg})\end{array}$ & Route & $\mathrm{n}$ & Male & $\begin{array}{l}\text { Gestational } \\
\text { age }\end{array}$ & $\begin{array}{l}\text { Birth } \\
\text { weight }\end{array}$ & Route \\
\hline Aly et al & Randomized & 12 & $\begin{array}{l}8 \\
(67)\end{array}$ & $32.9 \pm 1.6$ & $1.9 \pm 0.5$ & oral & 9 & $\begin{array}{l}4 \\
(44)\end{array}$ & $31.2 \pm 2.5$ & $\begin{array}{l}1.5 \pm \\
0.4\end{array}$ & IV \\
\hline Chan et al & Retrospective & 43 & $\begin{array}{l}20 \\
(47)\end{array}$ & $28.8 \pm 2.9$ & $1.1 \pm 0.4$ & IV & 52 & $\begin{array}{l}28 \\
(54)\end{array}$ & $28.4 \pm 3.2$ & $\begin{array}{l}1.1 \pm \\
0.5\end{array}$ & IV \\
\hline Fanos et al & Retrospective & 20 & $\begin{array}{l}14 \\
(70)\end{array}$ & $26.3 \pm 2.4$ & $0.9 \pm 0.3$ & IV & 20 & $\begin{array}{l}12 \\
(60)\end{array}$ & $26.5 \pm 2.2$ & $0.9 \pm 0.2$ & IV \\
\hline Hammerman et & Randomized & 32 & 14 & $27.8 \pm 2.6$ & $1.1 \pm 0.4$ & IV & 31 & 18 & $27.8 \pm 2.8$ & $1.1 \pm$ & IV \\
\hline
\end{tabular}




\section{Cureus}

al

$(44)$

Heo et al

Retrospective 22

(41)

$32.0 \pm 4.0$

$26.3 \pm 2.0$

Katakam et al

Retrospective 57

Randomized $94 \quad 50$

Lago et al

Lee et al

Linder et al

Mosca et al

Randomized

(50)

$29.0 \pm 1.2$

Patel et al

Patel et al

Pezzati et al

Randomized

Pourarian et al

Randomized

107

(70)

$31.3 \pm 4.4$

Retrospective 70

al

Su et al

Randomized 6034

$25.3 \pm 1.5$

Su et al

Van Overmeire et al

Van Overmeire et al

Yadav et al

Randomized 48

$48 \quad 26$

(54)

$29.7 \pm 3.2$

Yang et al

Retrospective 2

9

$26.7 \pm 1.0$

Chotigeat et al

Randomized 15

$15 \begin{aligned} & 8 \\ & (53)\end{aligned}$

$30.8 \pm 2.3$

(58)

0.5

$1.7 \pm 0.7 \quad$ oral $\quad 27 \begin{aligned} & 13 \\ & (41)\end{aligned} \quad 31.0 \pm 3.4$

$1.6 \pm$

0.6

IV

$1.0 \pm 0.3 \quad$ IV

65

$27.3 \pm 3.3$

$1.1 \pm$ 0.5

IV

$1.1 \pm 0.4 \quad$ IV

8144

$29.0 \pm 3.0$

$1.2 \pm$ 0.4

IV

$1.1 \pm 0.2 \quad$ oral $\quad 88$

43

$28.9 \pm 2.2$

$1.1 \pm$ 0.2

IV

$\begin{array}{lllllll}22 & 0.3 \quad \text { IV } \quad 46 & \begin{array}{l}22 \\ (48)\end{array} & 27.1 \pm 2.8 & 0.9 \pm & 0.3 & \text { IV }\end{array}$

$1.0 \pm 0.3 \quad$ IV $\quad 8$

(63)

$27.8 \pm 1.5$

$0.9 \pm$ 0.2

IV

IV

$1.2 \pm 0.7 \quad$ IV $\quad 15$

7
$(47)$

$26.7 \pm 2.0$

$0.9 \pm$ 0.3

IV

$1.2 \pm 0.4 \quad$ IV

$29.5 \pm 2.6$

$1.3 \pm$

IV

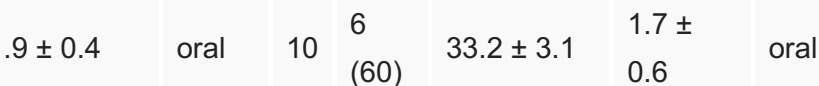
$\begin{array}{llllll}1.0 \pm 0.3 \quad \text { oral } \quad 54 & \begin{array}{l}27 \\ (50)\end{array} & 27.0 \pm 2.3 & 1.0 \pm & 0.3 & \text { IV }\end{array}$

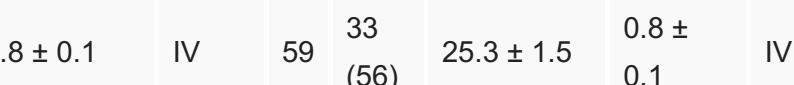

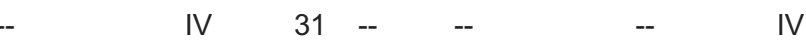

$1.3 \pm 0.5 \quad$ IV $\quad 20--28.7 \pm 1.9-1.2 \pm$

IV

0.4

$\begin{array}{lllllll}1.2 \pm 0.4 \quad \text { IV } \quad 74 & -- & 29.0 \pm 2.1 & 1.2 \pm & 0.4\end{array} \quad$ IV

$4+0.5 \quad$ oral $\quad 35-17 \quad 30.3 \pm 3.1-1.4 \pm$ oral

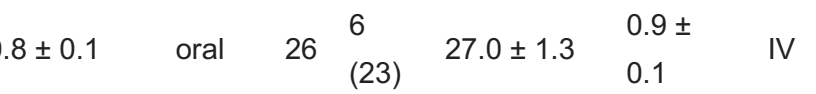
$1.4 \pm$
0.4 $\quad$ IV

\section{TABLE 1: Study characteristics}

Study characteristics 


\section{Cureus}

\section{Patient characteristics}

A total of 1,583 patients were included in this pooled analysis. Of the 22 studies, 14 compared IV ibuprofen to IV indomethacin with 552 patients in the ibuprofen group and 516 in the indomethacin group; six studies compared oral ibuprofen to IV indomethacin with 193 patients in the ibuprofen group and 219 in the indomethacin group; and two studies compared oral ibuprofen to oral indomethacin with 58 patients in the ibuprofen group and 45 in the indomethacin group. Additional data regarding patient characteristics can be found in Table 1 .

\section{PAD closure}

Fourteen studies were pooled comparing PAD closure with IV ibuprofen and IV indomethacin with a total of 552 patients having received ibuprofen and 16 having received indomethacin. Significant heterogeneity was not present (chi-squared $=6.09, \mathrm{p}=0.87, \mathrm{I}^{2}=0 \%$ ) so a fixed-effects model was used. There was no statistically significant difference in PAD closure with IV ibuprofen when compared to IV indomethacin (odds ratio 1.07, 95\% confidence interval 0.81 to 1.43) (Figure 2).

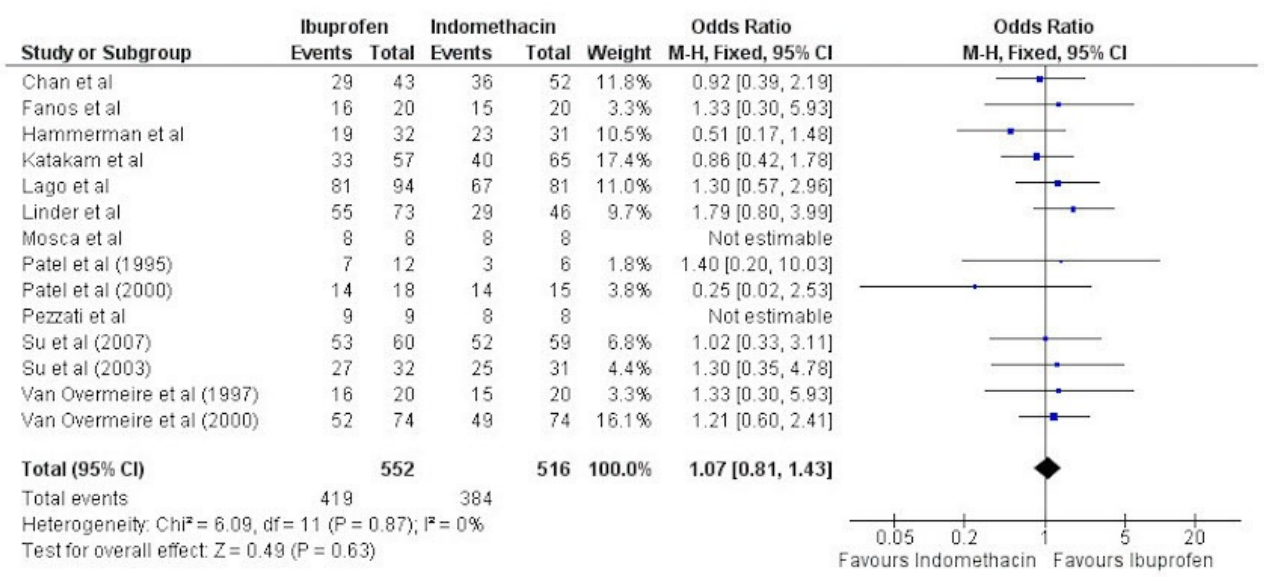

\section{FIGURE 2: Forest plot}

Forest plot comparing PAD closure between intravenous ibuprofen and intravenous indomethacin

Six studies were pooled comparing PAD closure with oral ibuprofen and IV indomethacin with 193 patients having received ibuprofen and 219 having received indomethacin. A fixed-effects model was used as significant heterogeneity was not present (chi-squared $=0.76, \mathrm{p}=0.51, \mathrm{I}^{2}=0 \%$ ). There was no statistically significant difference in PAD closure with oral ibuprofen when compared to IV indomethacin (odds ratio 0.76 , 95\% confidence interval 0.50 to 1.18) (Figure 3). 


\section{Cureus}

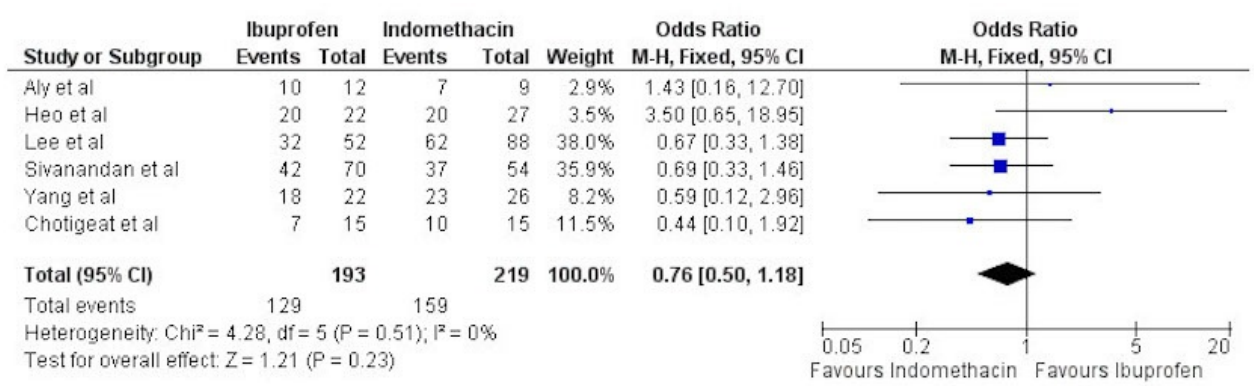

\section{FIGURE 3: Forest plot}

Forest plot comparing PAD closure between oral ibuprofen and intravenous indomethacin

Two studies contained data comparing PAD closure with oral ibuprofen and oral indomethacin with 58 patients in the ibuprofen group and 45 in the indomethacin group. Significant heterogeneity was not present (chi-squared=0.45, $\mathrm{p}=0.50, \mathrm{I}^{2}=0 \%$ ) so a fixed-effects model was used. There was no statistically significant difference between oral ibuprofen and oral indomethacin in respect to PAD closure (odds ratio $0.90,95 \%$ confidence interval 0.40 to 2.00 ) (Figure 4).

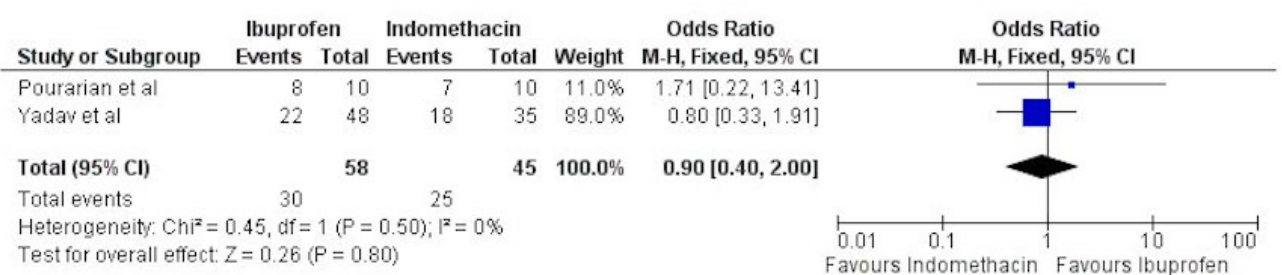

\section{FIGURE 4: Forest plot}

Forest plot comparing PAD closure between oral ibuprofen and oral indomethacin

\section{Need for surgical ligation}

Data regarding the need for surgical ligation comparing IV ibuprofen and IV indomethacin was available from nine studies with 418 patients having received ibuprofen and 417 having received indomethacin. A fixed-effects model was used as there was no significant heterogeneity (chi-squared $3.69, \mathrm{p}=0.88, \mathrm{I}^{2}=0 \%$ ). No statistically significant difference was noted in the need for surgical ligation between IV ibuprofen and IV indomethacin (odds ratio $0.86,95 \%$ confidence interval 0.58 to 1.28$)$.

Four studies compared need for surgical ligation in 159 patients having received oral ibuprofen and 183 having received IV indomethacin. A fixed-effects model was used as there was not significant heterogeneity (chi-squared $=2.47, \mathrm{p}=0.48, \mathrm{I}^{2}=0 \%$ ). The need for surgical ligation did not statistically significantly differ between oral ibuprofen and IV indomethacin (odds ratio 


\section{Death in first month of life}

A total of nine studies compared death in the first 30 days of life between 485 patients having received IV ibuprofen and 459 having received IV indomethacin. Significant heterogeneity was not present (chi-squared=11.24, $\mathrm{p}=0.19, \mathrm{I}^{2}=29 \%$ ). There was no statistically significant difference between those having received IV indomethacin and those having received IV ibuprofen (odds ratio $1.03,95 \%$ confidence interval 0.67 to 1.58 ).

\section{Necrotizing enterocolitis}

Eight studies compared the occurrence of necrotizing enterocolitis between IV ibuprofen and IV indomethacin with 412 and 413 patients in the two groups, respectively. No significant heterogeneity was present so a fixed-effects model was used (chi-squared=4.40, $\mathrm{p}=0.73, \mathrm{I}^{2}=0 \%$ ). No statistically significant difference in necrotizing enterocolitis was noted between IV ibuprofen and IV indomethacin (odds ratio 0.97, 95\% confidence interval 0.63 to 1.50 ).

Data regarding necrotizing enterocolitis comparing oral ibuprofen and IV indomethacin was available from five studies with 181 patients having received ibuprofen and 210 having received indomethacin. A fixed effects model was used as there was no significant heterogeneity (chisquared=3.93, $\mathrm{p}=0.42, \mathrm{I}^{2}=0$ ). No statistically significant difference was noted in necrotizing enterocolitis between oral ibuprofen and IV indomethacin (odds ratio $0.60,9 \%$ confidence interval 0.30 to 1.24$)$.

\section{Gastrointestinal bleeding}

Four studies compared the occurrence of gastrointestinal bleeding between 155 patients having received IV ibuprofen and 162 patients having received IV indomethacin. Significant heterogeneity was not present so a fixed-effects model was utilized. No statistically significant difference was noted in gastrointestinal bleeding between IV ibuprofen and IV indomethacin (odds ratio $1.40,95 \%$ confidence interval 0.73 to 2.69 ).

A total of three studies compared gastrointestinal bleeding between oral ibuprofen and IV indomethacin with 144 and 169 patients in each group, respectively. A fixed-effects model was used as significant heterogeneity was not present (chi-squared=2.75, $\mathrm{p}=0.25, \mathrm{I}^{2}=27 \%$ ). A statistically significant difference in gastrointestinal bleeding was not found between oral ibuprofen and IV indomethacin (odds ratio 0.62 , 95\% confidence interval 0.31 to 1.27 ).

\section{Intestinal perforation}

Seven studies compared intestinal perforation in 380 patients having received IV ibuprofen and 382 patients having received IV indomethacin. Significant heterogeneity was not present so a fixed-effects model was used (chi-squared=3.09, $\mathrm{p}=0.80, \mathrm{I}^{2}=0 \%$ ). A statistically significant difference in intestinal perforation was not noted between IV ibuprofen and IV indomethacin (odds ratio 1.09 , 95\% confidence interval 0.54 to 2.20 ).

\section{Bronchopulmonary dysplasia in the first month of life}

Data regarding the frequency of bronchopulmonary dysplasia in the first month of life was pooled from six studies with 323 patients having received IV ibuprofen and 317 having received IV indomethacin. A fixed-effects model was used as there was no significant heterogeneity noted (chi-squared $=5.69, \mathrm{p}=0.34, \mathrm{I}^{2}=12 \%$ ). No statistically significant difference was noted in 
bronchopulmonary dysplasia in the first month of life between IV ibuprofen and IV indomethacin (odds ratio $1.09,95 \%$ confidence interval 0.77 to 1.54 ).

A total of four studies were pooled to compare the frequency of bronchopulmonary dysplasia in the first month of life between 120 patients having received oral ibuprofen and 122 having received IV indomethacin. Significant heterogeneity was not present so a fixed-effects model was used (chi-squared $=3.35, \mathrm{p}=0.34, \mathrm{I}^{2}=10 \%$ ). No statistically significant difference in the frequency of bronchopulmonary dysplasia in the first month of life was noted between oral ibuprofen and IV indomethacin (odds ratio 0.80 , 95\% confidence interval 0.47 to 1.36 ).

\section{Grade $\mathbf{3}$ or $\mathbf{4}$ intraventricular hemorrhage}

Seven studies were pooled to compare the frequency of Grade 3 or 4 intraventricular hemorrhage between 392 patients having received IV ibuprofen and 393 patients having received IV indomethacin. A fixed-effects model was used as no significant heterogeneity was noted (chi-squared $=5.66, \mathrm{p}=0.46, \mathrm{I}^{2}=0 \%$ ). No significant difference was noted in Grade 3 or 4 intraventricular hemorrhage between IV ibuprofen and IV indomethacin (odds ratio 0.79, 95\% confidence interval 0.47 to 1.31 ).

\section{Change in creatinine}

A total of seven studies were pooled to compare changes in serum creatinine after attempted treatment between 345 patients having received IV ibuprofen and 310 patients having received IV indomethacin. Significant heterogeneity was present so a random-effects model was fixed (chi-squared=1244.00, $\mathrm{p}<0.001, \mathrm{I}^{2}=100 \%$ ). A statistically significant difference in change in creatinine was present and favored ibuprofen (mean difference -0.08, 95\% confidence interval 0.16 to 0.00 ) (Figure 5).

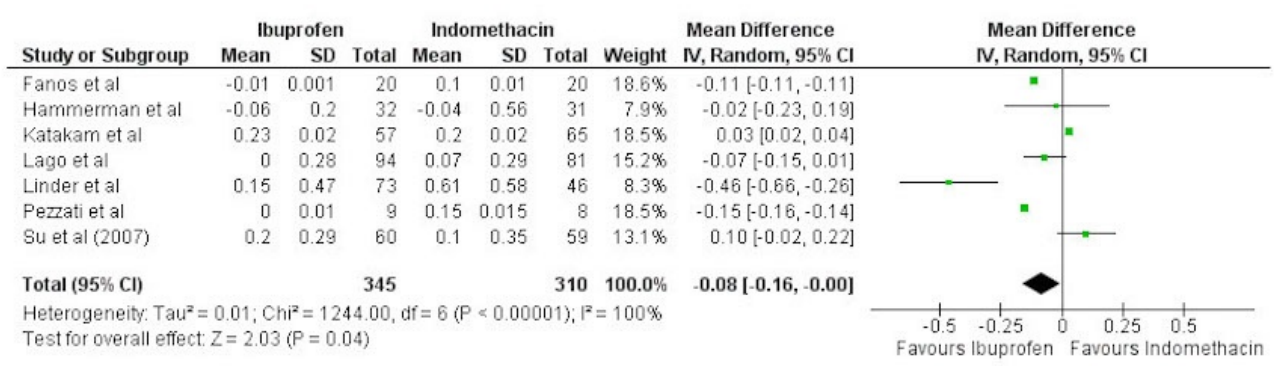

\section{FIGURE 5: Forest plot}

Forest plot comparing change in creatinine between intravenous ibuprofen and intravenous indomethacin

Five studies were pooled to compare changes in creatinine between 141 patients having received oral ibuprofen and 131 patients having received IV indomethacin. A fixed-effects model was used as no significant heterogeneity was present (chi-squared=4.09, p=0.39, $\mathrm{I}^{2}=2 \%$ ). No significant difference was noted between oral ibuprofen and IV indomethacin in regards to change in creatinine (mean difference -0.03 , 95\% confidence interval -0.11 to 0.05 ) (Figure 6). 


\section{Cureus}

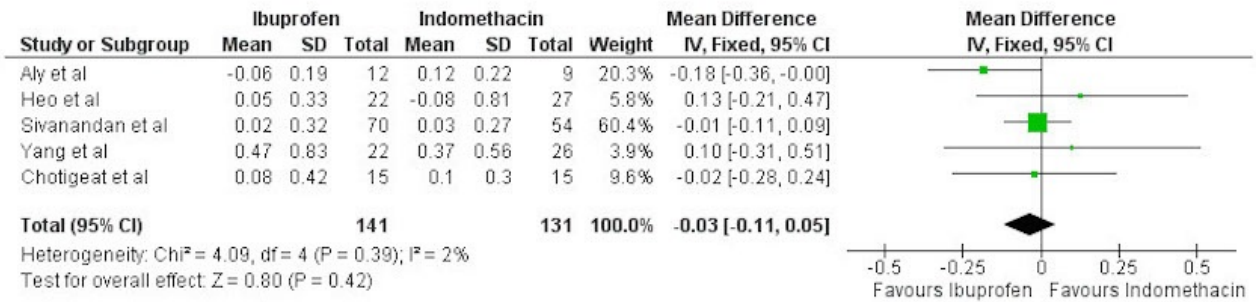

\section{FIGURE 6: Forest plot}

Forest plot comparing change in creatinine between oral ibuprofen and intravenous indomethacin

Data regarding change in creatinine between oral ibuprofen and oral indomethacin was available from two studies with 58 and 45 patients in the groups, respectively. A fixed-effects model was used as there was no significant heterogeneity present (chi-squared $=0.22, p=0.64$, $\left.\mathrm{I}^{2}=0 \%\right)$. A statistically significant difference in change in creatinine was present, favoring oral ibuprofen (mean difference $-0.10,95 \%$ confidence interval -0.13 to -0.07 ) (Figure 7 ).

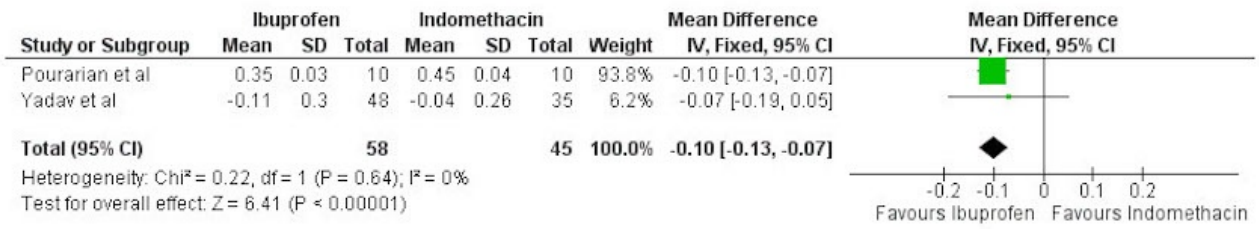

\section{FIGURE 7: Forest plot}

Forest plot comparing change in creatinine between oral ibuprofen and oral indomethacin

\section{Discussion}

This pooled analysis of 22 studies with 1,582 patients demonstrates that both ibuprofen and indomethacin are effective for PAD closure. Route of administration does not seem to affect efficacy and even oral ibuprofen seems to be equally effective as IV indomethacin. There was no difference in the incidence of surgical ligation between those given indomethacin and those given ibuprofen.

In regards to safety, there was no difference in the incidence of adverse outcomes, such as death in the first month of life, necrotizing enterocolitis, gastrointestinal bleeding, intestinal perforation, bronchopulmonary dysplasia in the first month of life, and Grade 3 or 4 intraventricular hemorrhage. There was a smaller increase in serum creatinine after attempted treatment associated with IV ibuprofen when compared to IV indomethacin and when oral ibuprofen was compared to IV indomethacin.

Previous studies have demonstrated a similar difference between ibuprofen and indomethacin 
in regards to change in creatinine $[12,18,22]$. Other studies have demonstrated that ibuprofen has less potential of adverse effects on renal function when compared to indomethacin using other endpoints, such as urine output as well as oliguria, which were not included in the current analysis due to the relatively low patient number in comparison to other endpoints. Urine output has been demonstrated to be higher in infants receiving ibuprofen rather than indomethacin for PAD closure.

The AD consists of an outer medial layer that consists of circumferential smooth muscle fibers supplied by thin-walled vasa vasorum while the inner medial layer is avascular and consists of longitudinal smooth muscle fibers. The internal elastic lamina separates this medial layer from the intima which is thin until the final end of gestation at which point it thickens. This is in contrast to the main pulmonary artery and the aorta which are comprised of circumferential elastic fibers [32].

The $\mathrm{AD}$ facilitates the passage of approximately $30 \%$ to $45 \%$ of the combined ventricular output in the human fetus, allowing for much of the blood to bypass the fetal lungs, which are immature and fluid filled, thus allowing them to complete development without the burden of a large amount of blood flow [33-35]. Since gas exchange occurs in the placenta, the fetal lungs require blood only for their own oxygenation and, therefore, do not require the entirety of the right ventricular output. The proportion of cardiac output that traverses the $\mathrm{AD}$ may presumably vary based on the amount of blood that is shunting at the atrial level via the foramen ovale, with the increased atrial level shunting leading to a decreased proportion of blood flow through the $\mathrm{AD}$. It has been demonstrated that blood with a higher oxygen content in the inferior vena cava has a higher flow velocity, which facilitates streaming of this blood preferentially through the foramen ovale. This then allows for more oxygenated blood to reach the left ventricle to be pumped to the coronary arteries and head and neck vessels via the ascending aorta. While this occurs, less oxygenated blood streams preferentially into the right ventricle and into the $\mathrm{AD}$ or fetal lungs [36-38].

While the specific mechanisms that mediate fetal patency of the AD are not entirely elucidated, several have been proposed, which include low partial pressure of oxygen, maternal prostaglandins, adenosine, and nitric oxide production [39-46]. Since these factors promote AD patency, it thus becomes intuitive that the converse of these would result in the closure of the ductus arteriosus which in fact is seen postnatally. The AD remains open in humans for approximately 24 to 48 hours after birth and will often have bi-directional flow initially, followed by a left to right shunt before it closes. In the immediate postnatal period when pulmonary vascular resistance is still elevated, a systolic shunt from the pulmonary artery to the descending aorta is present while, in diastole, a shunt from the aorta to the pulmonary artery is present. Once pulmonary vascular resistance has decreased further, the shunt becomes purely left to right. While the $\mathrm{AD}$ is closing, it is still reactive to certain mediators, such as the pressure of oxygen, and this decrease in the partial pressure of oxygen will lead to potential widening of the $\mathrm{AD}$ and an increase in pulmonary vascular resistance with the potential return of right to left shunting. Closure of the $\mathrm{AD}$ is found in $44 \%$ of full-term infants by 24 hours, $88 \%$ by 48 hours, and $100 \%$ by 72 hours [47].

Thickening of the intimal layer and the resulting decrease in luminal diameter facilitates closure of the AD. While this thickening occurs throughout gestation, it is particularly marked after birth. The internal elastic lamina also begins to fragment and allow smooth muscle and endothelial cell proliferation. Eventually, the AD constricts to a point where hypoxia develops. The vasa vasorum provides blood to the adventitia while the intima and the media receive blood directly from the lumen. Thus, hypoxia ensues as the AD constricts decreases flow to the media. With this hypoxia comes apoptosis and eventual fibrous tissue deposition, which completes the AD closure. In the former phases of this process where smooth muscle constriction and intimal thickening is occurring, the process may be reversible. Once the fibrous 
deposition has occurred, however, the process is no longer reversible [48].

Oxygen clearly plays a role in $\mathrm{AD}$ closure. Studies have demonstrated that increased partial pressure of oxygen resulted in $\mathrm{AD}$ closure [49-50]. This, however, only appears to be the response in late gestation as even exceedingly high partial pressures of oxygen do not induce constriction of the $\mathrm{AD}$ at earlier gestation. The mechanism behind this is unclear as $\mathrm{AD}$ constriction does occur with other agents, such as calcium and acetylcholine [50]. Studies demonstrating the need for an intact cytochrome oxiADse system for oxygen to produce $\mathrm{AD}$ constriction have raised the possibility that perhaps oxygen directly causes smooth muscle contraction. It has been hypothesized that perhaps oxygen binding to the cytochrome p450 hemoprotein may lead to membrane depolarization with calcium influx, which then leads to muscle contraction. It has also been suggested that perhaps oxygen-sensitive potassium channels in smooth muscle may cause depolarization and activation of voltage-sensitive calcium channels with subsequent smooth muscle contraction [51-52]. Other studies have also implicated the Rho-kinase system as well as endothelin-1 release as mechanisms by which oxygen may play a role in AD closure [53-54]. Additionally, the partial pressure of oxygen required to induce constriction has also been noted to decrease with increasing gestational age [50].

Nitric oxide, a potent vasodilator, is produced by endothelial cells utilizing the nitric oxide synthase. Nitric oxide synthase has been found in the $\mathrm{AD}$ and its surrounding endothelial tissue. Specific nitric oxide synthase inhibitors have also been demonstrated to be ineffective in antagonism of this system in the $\mathrm{AD}$ at low partial pressures of oxygen, which may also explain the mechanism by which oxygen affects $\mathrm{AD}$ constriction. Additionally, this system thus facilitates AD closure at a later gestational age [44].

Other vasoactive agents, such as adenosine, acetylcholine, bradykinin, and norepinephrine, have also been demonstrated to lead to AD constriction and have synergistic effects to the AD's response to oxygen. The absence of any of these agents, however, does not impair oxygenmediated $\mathrm{AD}$ constriction. The precise role of these agents is not clearly defined [46, 55].

Of particular importance is the effect of prostaglandins on AD closure as both ibuprofen and indomethacin target the prostaglandin pathway. Cyclooxygenase is the enzyme responsible for synthesizing prostaglandins from arachidonic acid [56]. There are two varieties of this enzyme and both have been found to participate in prostaglandin synthesis in the wall of the $\mathrm{AD}$, resulting in both prostaglandin $\mathrm{E}_{2}$ and prostaglandin $\mathrm{I}_{2}$. While prostaglandin $\mathrm{I}_{2}$ is more abundant, prostaglandin $\mathrm{E}_{2}$ is more potent with the $\mathrm{AD}$ being nearly 1,000 times more sensitive to prostaglandin $\mathrm{E}_{2}$, which is believed to play a vital role in maintaining the $\mathrm{AD}$ during fetal life [39]. In fact, prostaglandin $E_{2}$ levels are higher in the fetus than in the mother with levels falling rapidly after birth, such that adult levels are reached by three hours of life [42]. What is particularly intriguing is that reductions in pulmonary blood flow, as may be seen secondary to lung disease or pulmonary vasoconstriction, can delay this fall in prostaglandin $\mathrm{E}_{2}$ levels since the lungs are the site of its metabolism. The partial pressure of oxygen does not impact the effect of prostaglandin $\mathrm{E}_{2}$ on $\mathrm{AD}$ constriction. Premature infants have both continued production of prostaglandin $\mathrm{E}_{2}$ but also an increased response to it [57].

These findings regarding the effect of prostaglandins on $\mathrm{AD}$ closure have important clinical implications. Both ibuprofen and indomethacin are nonselective cyclooxygenase inhibitors and also inhibit the synthesis of prostaglandin $\mathrm{E}_{2}$. Thus, understanding of the previously mentioned characteristics of the AD make the use of cyclooxygenase inhibitors intuitive in the setting of premature infants with PAD [58]. 
This study demonstrates that ibuprofen and indomethacin are equally effective in closing the PAD with ibuprofen having a renoprotective effect with some dosage routes. This current manuscript also reviews the embryology and physiology of the AD. This analysis, however, is not without its limitations. Pooled data is study-level rather than patient-level, thus limiting the opportunity for specific meta-regression. Additionally, some endpoints had significant heterogeneity. Even those endpoints without quantitative endpoints may have qualitative heterogeneity. Additionally, no cost analysis could be conducted. There was a small number of studies available to pool in regards to the comparison of the oral ibuprofen and oral indomethacin as well.

\section{Conclusions}

Ibuprofen and indomethacin are both equally effective in closure of the PAD in preterm infants. Adverse effects associated were not found to differ between the two, although ibuprofen was associated with a lower increase in creatinine.

\section{Additional Information \\ Disclosures}

Human subjects: All authors have confirmed that this study did not involve human participants or tissue. Animal subjects: All authors have confirmed that this study did not involve animal subjects or tissue. Conflicts of interest: In compliance with the ICMJE uniform disclosure form, all authors declare the following: Payment/services info: All authors have declared that no financial support was received from any organization for the submitted work. Financial relationships: All authors have declared that they have no financial relationships at present or within the previous three years with any organizations that might have an interest in the submitted work. Other relationships: All authors have declared that there are no other relationships or activities that could appear to have influenced the submitted work.

\section{References}

1. Auld PA: Delayed closure of the ductus arteriosus . J Pediatr. 1966, 69:61-66. 10.1016/S00223476(66)80362-1

2. Danilowicz D, Rudolph AM, Hoffman JI: Delayed closure of the ductus arteriosus in premature infants. Pediatrics. 1966, 37:74-78.

3. Reller MD, Colasurdo MA, Rice MJ, McDonald RW: The timing of spontaneous closure of the ductus arteriosus in infants with respiratory distress syndrome. Am J Cardiol. 1990, 66:75-78. 10.1016/0002-9149(90)90739-N

4. Evans N: Preterm patent ductus arteriosus: should we treat it? . J Paediatr Child Health. 2012, 48:753-758. 10.1111/j.1440-1754.2012.02542.x

5. Clyman RI, Couto J, Murphy GM: Patent ductus arteriosus: are current neonatal treatment options better or worse than no treatment at all?. Semin Perinatol. 2012, 36:123-129. 10.1053/j.semperi.2011.09.022

6. Meyer S: PDA in neonates--Please Doctor Act individually! Acta Paediatr. 2012, 101:e145. 10.1111/j.1651-2227.2012.02608.x

7. Schena F, Ciarmoli E, Mosca F: Patent ductus arteriosus: wait and see? J Matern Fetal Neonatal Med. 2011, 24:2-4. 10.3109/14767058.2011.607716

8. Abdel-Hady H, Nasef N, Shabaan AE, Nour I: Patent ductus arteriosus in preterm infants: do we have the right answers?. Biomed Res Int. 2013, 2013:676192. 10.1155/2013/676192

9. Noori S: Pros and cons of patent ductus arteriosus ligation: hemodynamic changes and other morbidities after patent ductus arteriosus ligation. Semin Perinatol. 2012, 36:139-145. 10.1053/j.semperi.2011.09.024

10. Aly H, Lotfy W, Badrawi N, Ghawas M, Abdel-Meguid IE, Hammad TA: Oral Ibuprofen and ductus arteriosus in premature infants: a randomized pilot study. Am J Perinatol. 2007, 24:267-270. 10.1055/s-2007-976550 
11. Chan NM, Law CW, Kwan KF: Ibuprofen versus indomethacin treatment of patent ductus arteriosus: comparative effectiveness and complications. Hong Kong Med J. 2014, 20:205-212. 10.12809/hkmj134080

12. Fanos V, Benini D, Verlato G, Errico G, Cuzzolin L: Efficacy and renal tolerability of ibuprofen vs. indomethacin in preterm infants with patent ductus arteriosus. Fundam Clin Pharmacol. 2005, 19:187-193. 10.1111/j.1472-8206.2004.00314.X

13. Hammerman C, Shchors I, Jacobson S, Schimmel MS, Bromiker R, Kaplan M, Nir A: Ibuprofen versus continuous indomethacin in premature neonates with patent ductus arteriosus: is the difference in the mode of administration?. Pediatr Res. 2008, 64:291-297. 10.1203/PDR.0b013e31817d9bb0

14. Heo MJ, Lee OS, Lim SC: Comparative evaluation for the use of oral ibuprofen and intravenous indomethacin in Korean infants with patent ductus. Arch Pharm Res. 2012, 35:1673-1683. 10.1007/s12272-012-0919-8

15. Katakam LI, Cotten CM, Goldberg RN, Dang CN, Smith PB: Safety and effectiveness of indomethacin versus ibuprofen for treatment of patent ductus arteriosus. Am J Perinatol. 2010, $27: 425-429.10 .1055 / \mathrm{s}-0029-1243371$

16. Lago P, Bettiol T, Salvadori S, Pitassi I, Vianello A, Chiandetti L, Saia OS: Safety and efficacy of ibuprofen versus indomethacin in preterm infants treated for patent ductus arteriosus: a randomised controlled trial. Eur J Pediatr. 2002, 161:202-207. 10.1007/s00431-002-0915-y

17. Lee CH, Chen HN, Tsao LY, Hsiao CC, Lee ML: Oral ibuprofen versus intravenous indomethacin for closure of patent ductus arteriosus in very low birth weight infants. Pediatr Neonatol. 2012, 53:346-353. 10.1016/j.pedneo.2012.08.011

18. Linder N, Bello R, Hernandez A, Rosen C, Birk E, Sirota L, Pushkov Y, Klinger G: Treatment of patent ductus arteriosus: indomethacin or ibuprofen?. Am J Perinatol. 2010, 27:399-404. $10.1055 /$ s-0029-1243315

19. Mosca F, Bray M, Lattanzio M, Fumagalli M, Tosetto C: Comparative evaluation of the effects of indomethacin and ibuprofen on cerebral perfusion and oxygenation in preterm infants with patent ductus arteriosus. J Pediatr. 1997, 131:549-554. 10.1016/S0022-3476(97)70060-X

20. Patel J, Marks KA, Roberts I, Azzopardi D, Edwards AD: Ibuprofen treatment of patent ductus arteriosus. Lancet. 1995, 346:255. 10.1016/S0140-6736(95)91304-1

21. Patel J, Roberts I, Azzopardi D, Hamilton P, Edwards AD: Randomized double-blind controlled trial comparing the effects of ibuprofen with indomethacin on cerebral hemodynamics in preterm infants with patent ductus arteriosus. Pediatr Res. 2000, 47:36-42. 10.1203/00006450-200001000-00009

22. Pezzati M, Vangi V, Biagiotti R, Bertini G, Cianciulli D, Rubaltelli FF: Effects of indomethacin and ibuprofen on mesenteric and renal blood flow in preterm infants with patent ductus arteriosus. J Pediatr. 1999, 135:733-738. 10.1016/S0022-3476(99)70093-4

23. Pourarian Sh, Pishva N, Madani A, Rastegari M: Comparison of oral ibuprofen and indomethacin on closure of patent ductus arteriosus in preterm infants. East Mediterr Health J. 2008, 14:360-365.

24. Sivanandan S, Bali V, Soraisham AS, Harabor A, Kamaluddeen M: Effectiveness and safety of indomethacin versus ibuprofen for the treatment of patent ductus arteriosus in preterm infants. Am J Perinatol. 2013, 30:745-750. 10.1055/s-0032-1332800

25. Su BH, Lin HC, Chiu HY, Hsieh HY, Chen HH, Tsai YC: Comparison of ibuprofen and indometacin for early-targeted treatment of patent ductus arteriosus in extremely premature infants: a randomised controlled trial. Arch Dis Child Fetal Neonatal Ed. 2008, 93:F94-99. 10.1136/adc.2007.120584

26. Su PH, Chen JY, Su CM, Huang TC, Lee HS: Comparison of ibuprofen and indomethacin therapy for patent ductus arteriosus in preterm infants. Pediatr Int. 2003, 45:665-670. 10.1111/j.1442-200X.2003.01797.x

27. van Overmeire B, Brus F, van Acker KJ, van der Auwera JC, Schasfoort M, Elzenga NJ, Okken A: Aspirin versus indomethacin treatment of patent ductus arteriosus in preterm infants with respiratory distress syndrome. Pediatr Res. 1995, 38:886-891. 10.1203/00006450-19951200000010

28. Van Overmeire B, Smets K, Lecoutere D, Van de Broek H, Weyler J, Degroote K, Langhendries JP: A comparison of ibuprofen and indomethacin for closure of patent ductus arteriosus . NEJM. 2000, 343:674-681. 10.1056/NEJM200009073431001

29. Yadav S, Agarwal S, Maria A, Dudeja A, Dubey NK, Anand P, Yadav DK: Comparison of oral 
ibuprofen with oral indomethacin for PDA closure in Indian preterm neonates: a randomized controlled trial. Pediatr Cardiol. 2014, 35:824-830. 10.1007/s00246-014-0861-2

30. Chotigeat U, Jirapapa K, Layangkool: A comparison of oral ibuprofen and intravenous indomethacin for closure of patent ductus arteriosus in preterm infants. J Med Assoc Thai. 2003, 86:S563-569.

31. Yang EM, Song ES, Choi YY: Comparison of oral Ibuprofen and intravenous indomethacin for the treatment of patent ductus arteriosus in extremely low birth weight infants. J Pediatr (Rio J). 2013, 89:33-39. 10.1016/j.jped.2013.02.006

32. Rudolph AM: Congenital Diseases of the Heart: Clinical-Physiological Considerations . Rudolph AM (ed): Wiley-Blackwell, Chichester, UK; Hoboken, NJ; 2009.

33. Mielke G, Benda N (2001: Cardiac output and central distribution of blood flow in the human fetus. Circulation. 2001, 103:1662-1668. 10.1161/01.CIR.103.12.1662

34. Sutton MS, Groves A, MacNeill A, Sharland G, Allan L: Assessment of changes in blood flow through the lungs and foramen ovale in the normal human fetus with gestational age: a prospective Doppler echocardiographic study. Br Heart J. 1994, 71:232-237. 10.1136/hrt.71.3.232

35. Rasanen J, Wood DC, Weiner S, Ludomirski A, Huhta JC: Role of the pulmonary circulation in the distribution of human fetal cardiac output during the second half of pregnancy. Circulation. 1996, 94:1068-1073.

36. Bristow J, Rudolph AM, Itskovitz: A preparation for studying liver blood flow, oxygen consumption, and metabolism in the fetal lamb in utero. J Dev Physiol. 1981, 3:255-266.

37. Edelstone DI, Rudolph AM: Preferential streaming of ductus venosus blood to the brain and heart in fetal lambs. Am J Physiol. 1979, 237:H724-729.

38. Schmidt KG, Silverman NH, Rudolph AM: Assessment of flow events at the ductus venosusinferior vena cava junction and at the foramen ovale in fetal sheep by use of multimodal ultrasound. Circulation. 1996, 93:826-833. 10.1161/01.CIR.93.4.826

39. Clyman RI, Mauray F, Roman C, Rudolph AM: PGE2 is a more potent vasodilator of the lamb ductus arteriosus than is either PGI2 or 6 keto PGF1alpha. Prostaglandins. 1978, 16:259-264. 10.1016/0090-6980(78)90028-X

40. Clyman RI, Mauray F, Rudolph AM, Heymann MA: Age-dependent sensitivity of the lamb ductus arteriosus to indomethacin and prostaglandins. J Pediatr. 1980, 96:94-98. 10.1016/S0022-3476(80)80338-6

41. Clyman RI, Brett C, Mauray: Circulating prostaglandin E2 concentrations and incidence of patent ductus arteriosus in preterm infants with respiratory distress syndrome. Pediatrics. 1980, 66:725-729.

42. Clyman RI, Mauray F, Roman C, Rudolph AM, Heymann MA: Circulating prostaglandin E2 concentrations and patent ductus arteriosus in fetal and neonatal lambs. J Pediatr. 1980, 97:455-461. 10.1016/S0022-3476(80)80205-8

43. Clyman RI: Ductus arteriosus: developmental response to endogenous prostaglandins, oxygen, and indomethacin. Adv Prostaglandin Thromboxane Res. 1980, 7:887-890.

44. Clyman RI, Waleh N, Black SM, Riemer RK, Mauray F, Chen YQ: Regulation of ductus arteriosus patency by nitric oxide in fetal lambs: the role of gestation, oxygen tension, and vasa vasorum. Pediatr Res. 1998, 43:633-644. 10.1203/00006450-199805000-00012

45. Momma K, Toyono M: The role of nitric oxide in dilating the fetal ductus arteriosus in rats . Pediatr Res. 1999, 46:311-315. 10.1203/00006450-199909000-00010

46. Mentzer RM Jr, Ely SW, Lasley RD, Mainwaring RD, Wright EM Jr, Berne RM: Hormonal role of adenosine in maintaining patency of the ductus arteriosus in fetal lambs. Ann Surg. 1985, 202:223-230. 10.1097/00000658-198508000-00013

47. Shiraishi H, Yanagisawa M: Bidirectional flow through the ductus arteriosus in normal newborns: evaluation by Doppler color flow imaging. Pediatr Cardiol. 1991, 12:201-205. 10.1007/BF02310566

48. Clyman RI, Chan CY, Mauray F, Chen YQ, Cox W, Seidner SR, Lord EM, Weiss H, Waleh N, Evans SM, Koch CJ: Permanent anatomic closure of the ductus arteriosus in newborn baboons: the roles of postnatal constriction, hypoxia, and gestation. Pediatr Res. 1999, 45:19-29. 10.1203/00006450-199901000-00005

49. Oberhänsli-Weiss I, Heymann MA, Rudolph AM, Melmon KL: The pattern and mechanisms of response to oxygen by the ductus arteriosus and umbilical artery. Pediatr Res. 1972, 6:693700. 10.1203/00006450-197207000-00001 
50. McMurphy DM, Heymann MA, Rudolph AM, Melmon KL: Developmental changes in constriction of the ductus arteriosus: responses to oxygen and vasoactive agents in the isolated ductus arteriosus of the fetal lamb. Pediatr Res. 1972, 6:231-238. 10.1203/00006450197204000-00004

51. Fay FS, Nair P, Whalen WJ: Mechanism of oxygen induced contraction of ductus arteriosus . Adv Exp Med Biol. 1977, 78:123-134. 10.1007/978-1-4615-9035-4_9

52. Coceani F, Hamilton NC, Labuc J, Olley PM: Cytochrome P 450-linked monooxygenase: involvement in the lamb ductus arteriosus. Am J Physiol. 1984, 246:H640-643.

53. Coceani F, Kelsey L, Seidlitz: Evidence for an effector role of endothelin in closure of the ductus arteriosus at birth. Can J Physiol Pharmacol. 1992, 70:1061-1064. 10.1139/y92-146

54. Kajimoto H, Hashimoto K, Bonnet SN, Haromy A, Harry G, Moudgil R, Nakanishi T, Rebeyka I, Thébaud B, Michelakis ED, Archer SL: Oxygen activates the Rho/Rho-kinase pathway and induces RhoB and ROCK-1 expression in human and rabbit ductus arteriosus by increasing mitochondria-derived reactive oxygen species: a newly recognized mechanism for sustaining ductal constriction. Circulation. 2007, 115:1777-1788.

10.1161/CIRCULATIONAHA.106.649566

55. Sawa R, Asakura H, Power GG: Changes in plasma adenosine during simulated birth of fetal sheep. J Appl Physiol (1985). 1991, 70:1524-1528.

56. Takahashi Y, Roman C, Chemtob S, Tse MM, Lin E, Heymann MA, Clyman RI: Cyclooxygenase-2 inhibitors constrict the fetal lamb ductus arteriosus both in vitro and in vivo. Am J Physiol Regul Integr Comp Physiol. 2000, 278:R1496-1505.

57. Clyman RI, Heymann MA, Rudolph AM: Ductus arteriosus responses to prostaglandin E1 at high and low oxygen concentrations. Prostaglandins. 1977, 13:219-223. 10.1016/00906980(77)90003-X

58. Clyman RI, Mauray F, Heymann MA, Rudolph AM: Ductus arteriosus: developmental response to oxygen and indomethacin. Prostaglandins. 1978, 15:993-998. 10.1016/00906980(78)90041-2 